\title{
The future of technologies for personalised medicine
}

\section{Alison Harvey ${ }^{1}$, Angela Brand ${ }^{2,4}$, Stephen T. Holgate ${ }^{2,5}$, Lars V. Kristiansen ${ }^{3}$, Hans Lehrach ${ }^{2,6}$, Aarno Palotie ${ }^{2,7,8}$ and Barbara Prainsack ${ }^{2,9}$}

\footnotetext{
${ }^{1}$ Finchley Court, Norwich, UK

${ }^{2}$ Scientific Committee for the ESF Forward Look on Personalised Medicine for the European Citizen, France

${ }^{3}$ Biomedical Sciences Unit, the European Science Foundation, Strasbourg, France

${ }^{4}$ Institute for Public Health Genomics, Maastricht University, Maastricht, Netherlands

${ }^{5}$ Faculty of Medicine, University of Southampton, Southampton, UK

${ }^{6}$ Max Planck Institute for Molecular Genetics, Berlin, DE, Germany

${ }^{7}$ Institute for Molecular Medicine Finland (FIMM), University of Helsinki, Helsinki, Finland

${ }^{8}$ The Wellcome Trust Sanger Institute, Cambridge, UK

${ }^{9}$ Brunel University, Uxbridge Middlesex, UK
}

Personalised medicine promises prediction, prevention and treatment of illness that is targeted to individuals' needs. New technologies for detailed biological profiling of individuals at the molecular level have been crucial in initiating the move to personalised medicine; further novel technologies will be necessary if the vision is to become a reality. We will need to develop new technologies to collect and analyse data in a way that is not just linear but integrated (understanding system level functioning) and dynamic (understanding system in flux). Key factors in the development of technologies for personalised medicine are standardisation, integration and harmonisation. For example, the tools and processes for data collection and analysis must be standardised across research sites. Research activity at different sites must be integrated to maximise synergies, and scientific research must be integrated with healthcare to ensure effective translation. There must also be harmonisation between scientific practices in different research sites, between science and healthcare and between science, healthcare and wider society, including the ethical and regulatory frameworks, the prevailing political and cultural ethos and the expectations of patients/citizens.

\section{Introduction}

Healthcare that extrapolates from population averages to treat or prevent disease in individuals by trial and error is becoming a thing of the past. Great hopes are being invested in 'personalised medicine' as a form of healthcare that is better tailored to individuals' needs. The vision is that healthcare will shift from a 'one size fits all' approach towards more targeted prediction, prevention and treatment of illness [1-5]. New techniques and technologies such as those that allow detailed biological profiling of individuals at the molecular level are one of the factors driving this shift. Further novel technologies will be required if the vision for a personalised medicine is to be realised in practice. In many contexts we have already started to move from treating diseases to treating individuals.

To understand the technological requirements for the further development of personalised medicine, the European Science Foundation (ESF) (http://www.esf.org) recently held a workshop as part of its Forward Look on Personalised Medicine for the European 
TABLE 1

What is required to turn more information into personalised medicine

\begin{tabular}{|c|c|c|}
\hline Research & Translation & Healthcare \\
\hline Standardisation & $\begin{array}{l}\text { New methodologies for evaluating } \\
\text { validity and utility }\end{array}$ & Easy to use diagnostic and monitoring tools \\
\hline
\end{tabular}

Quantification

Computing resources

Computing algorithms

Integration of data
Near-patient diagnostics to aid prescribing

Novel technologies for real time longitudinal monitoring of functional status
Citizen: towards more precise medicine for the diagnosis, treatment and prevention of disease. ${ }^{\text {a }}$ The ESF Forward Looks enable Europe's scientific community, in interaction with policy makers, to develop medium- to long-term views and analyses of future research developments with the aim of defining research agendas at national and European level. The overall aim of this ESF Forward Look is to analyse in a systematic way the complex and constantly moving field of personalised medicine to provide timely policy advice that will help prepare Europe for the likely changes in how society deals with health and disease. The workshop on technology brought together scientists, clinicians, public health experts and other stakeholders to discuss how technological developments and integration of existing and novel technologies may shape the longer term future of personalised medicine.

In asking participants to consider the longer term future of the technologies for personalised medicine, the brief was to focus on developments over the next 20 years. Consideration was given to how the existing technologies that are making personalised medicine possible might need to evolve (supply push). But attention (and imagination) was also devoted to thinking about possible

\footnotetext{
${ }^{a}$ The workshop participants were: Rudolf Aebersold (Swiss Federal Institute of Technology, Zurich, CH); Rolf Apweiler (Wellcome Trust Genome Campus Hinxton, Cambridge, UK); Ivano Bertini (University of Florence, Sesto Fiorentino, IT); Thomas Beyer (CMI-Experts GmbH, Zurich, CH); Patrick Boisseau (Commissariat à l'énergie atomique (CEA) - LETI, Grenoble, FR); Angela Brand (Maastricht University, Maastricht, NL); Carsten Carlberg (University of Eastern Finland, Kuopio, Fl); Falk Ehmann (European Medicines Agency (EMA), London, UK); Alex Faulkner (King's College London, London, UK); Ivo Gut (Centro Nacional de Análisis Genómico, Barcelona, ES); Adriano Henney (Obsidian Biomedical Consulting Ltd, Macclesfield, UK); Stuart Hogarth (King's College London, London, UK); Stephen T. Holgate (University of Southampton, Southampton, UK); Bärbel Hűsing (Fraunhofer ISI, Karlsruhe, DE); Gabriel P. Krestin (Erasmus Medical Center, Rotterdam, NL); Alexander Kühn (Max Planck Institute for Molecular Genetics, Berlin, DE); Lars V. Kristiansen (The European Science Foundation (ESF), Strasbourg, FR); Piret Kukk (Fraunhofer ISI, Karlsruhe, DE); Ulf Landegren (Uppsala University, Uppsala, SE); Pierre Legrain (Commissariat à I'Energie Atomique (CEA), Fontenay aux Roses, FR); Hans Lehrach (Max Planck Institute for Molecular Genetics, Berlin, DE); Daniel MacArthur (Wellcome Trust Genome Campus Hinxton, Cambridge, UK); John McGrath (King's College London, London, UK); Peter Mills (Nuffield Council on Bioethics, London, UK); Francesc Palau Martínez (Instituto Biomedicina Valencia, Valencia, ES); Aarno Palotie (Finnish Genome Centre, Helsinki, FI); Marisa Papaluca Amati (European Medicine Agency (EMA), London, UK); lain Patten (Medical and Scientific Writing Consultant, Valencia, ES); Barbara Prainsack (Brunel University, Uxbridge Middlesex, UK); Timothy Spector (King's College London, London, UK); Ralf Sudbrak (Max Planck Institute for Molecular Genetics, Berlin, DE); Ben Van Ommen (Nutrition and Food Research Institute (TNO), HE Zeist, NL); Stephen Williams (SomaLogic Inc., Boulder, USA); Kurt Zatloukal (Medical University of Graz, Graz, AT).
}

revolutionary developments, and the sorts of novel technologies and their interoperability that we might want to see in the future to realise the potential of personalised medicine (demand pull).

The insights generated at this workshop form the basis for this paper. We do not offer a comprehensive report of the discussions, but instead identify the key issues and themes that emerged. We present an account of the technologies that will be central to the realisation of personalised medicine and consider the conditions that will be necessary if those technologies are to be developed. As such, the paper sets out an agenda for the future of technologies for personalised medicine.

\section{The information requirements of personalised medicine}

'Personalised medicine' is not a precise term. In this paper, we use it in a broad sense that encompasses various approaches to tailoring healthcare. The medical profession will point out that it has always practised personalised medicine. Doctors will consider factors such as age, sex and family history in making a diagnosis, and will tailor treatment to the individual patient taking into account factors such as co-morbidities and attending to the patient's psycho-social, lifestyle and possibly family and economic circumstances. However, medical practice has, especially over the past 60 years or so, been under an increasing pressure to become more 'scientific' [6]. Although medical practice has arguably always been evidence-based (including evidence produced by narrative accounts, palpation, demographic and social factors concerning the patient, among others), what has changed is our understanding and use of evidence. The collection, processing and analysis of data have become more systematic, and often automated. Data are also more easily aggregated and mined. It is against this backdrop that the commitment to Evidence Based Medicine (EBM) as a central tenet of medical practice needs to be understood. EBM is 'the conscientious, explicit, and judicious use of current best evidence in making decisions about the care of individual patients' [7: 71], this evidence being encapsulated in the guidelines, standards, decision support tools and so on that have become an increasingly common resource in healthcare. The randomised controlled trial (RCT) has become the 'gold standard' for generation of best evidence in medical science [8], producing information on the optimum course of action for the 'average patient'. However, even this is being challenged on account of being conducted on highly selected and unrepresentative populations using endpoints more suited to physicians than patients. The current interest in personalised medicine is about personalising mostly the science of medicine (which, in turn, will have an effect 
TABLE 2

What is needed to make the move to personalised medicine possible

\begin{tabular}{|c|c|c|}
\hline Integration & Harmonisation & Resources \\
\hline Technical & Data & Machines \\
\hline Organisational & Communication & People and institutions \\
\hline Cultural: science and healthcare & Regulation & Funding and infrastructures \\
\hline \multicolumn{3}{|c|}{ Culture: biomedicine/public health and society } \\
\hline
\end{tabular}

on the personalisation of care). It promises to move beyond the generation of evidence on the optimal intervention for the 'average patient' towards producing scientific knowledge and 'individual evidence' of how best to treat the specific person on the basis that each individual has special characteristics of their disease moving medicine away from 'one size fits all' towards 'personalised interventions' (Tables 1 and 2).

We are already familiar with selecting antimicrobial drugs on the basis of infecting disease organism sensitivities. So is the case in the intact human in whom more personalised medicine has already started to become a reality, with the advent of pharmacogenomics. For example, research elucidating the variation in response to drugs at the genetic level is making it possible to determine who will or will not benefit from a particular therapy (e.g., in colorectal cancer, those in whom the tumour carries a mutation in the KRAS gene show no response to cetuximab and panitumumab), or who will respond adversely (e.g. presence of the HLA-B 5701 allele is associated with a hypersensitivity reaction to Abacavir, used in the treatment of AIDS) [9].

Pharmacogenomics personalises medicine to the extent that it stratifies patients with a specific disease into subgroups. This stratification results in a tailoring that is analogous to having a choice of small, medium or large, rather than one-size-fits-all; it is not the provision of a bespoke suit [Steven Rose, cited in 10: 5]. We are likely to see more tailoring through stratification, both through expansion of pharmacogenomics, and through research that, by elucidating the molecular basis of pathologies, is redefining what would once have been considered a single disease entity into different disease subtypes (as has revolutionised the treatment of leukaemias) or indeed different illnesses, re-categorising common complex disease into multiple rare diseases [11]. However, in parallel to the advancement of tailoring through stratification, there is also interest in progressing tailoring through individualisation $[1,3]$. This entails treating the person as unique, and as a whole. '-Omics' type analysis techniques, for example the sequencing of the complete genome and transcriptome of a tumour compared to the genome of the patient, can form the basis of computer models of the virtual patient, which can then be used to identify an individually optimised therapy. Systems biology approaches produce an integrated understanding of the organism's biological functioning as a complex and dynamic entity. The corresponding healthcare practices would take a holistic approach, assessing the person's individual biological make-up interacting with the environment and developing interventions to maximise wellbeing. Integrating information about the person's biological makeup, using tools such as gene pathway analyses and in silico modelling, would allow physicians to select the optimum therapy for that patient. It also promises to drive a renewed attention to preventive healthcare, enabling advice on disease-prevention strategies to be tailored to the specific individual (Fig. 1).

Whether stratified or individualised, personalised medicine with its promise of better, more precise treatment for individuals is premised on the availability of more information about individuals. There is demand for quantitative increase: more information, and more detailed information, about the biology of humans, derived from 'omics' data (genomics, epigenomics, transcriptomics, proteomics, metabolomics, among others) in combination with other sorts of data (biological imaging, physiological measurements, among others). There is also demand for qualitative increase: new types of data such as data on the person's environment (nutrients, the microbiome, toxin exposure, among others) are being seen as important for understanding biological functioning. More information is required in both the research setting, and in healthcare practice, and there must be an accepted process or processes for evaluating how knowledge generated in the former sphere of activity is applicable in the latter (Fig. 2).

If personalised medicine is to move from stratified medicine to a future with individualised medicine and individualised healthcare, as outlined in Fig. 1, then it will be necessary to generate different forms of information about biological functioning (Fig. 3). Bioscience research is becoming adept at producing 'linear' data, data on a specific feature of the organism (e.g. its genome or transcriptome or proteome) as a snapshot in time. Researchers have started to address how these different elements interact, integrating the data from different sources (to study the interplay between genetic and environmental variables for example), and so beginning to unpick the complexity of the organism's functioning as 'more than the sum of its parts' [12]. A further step would entail developing the technologies to understand how the

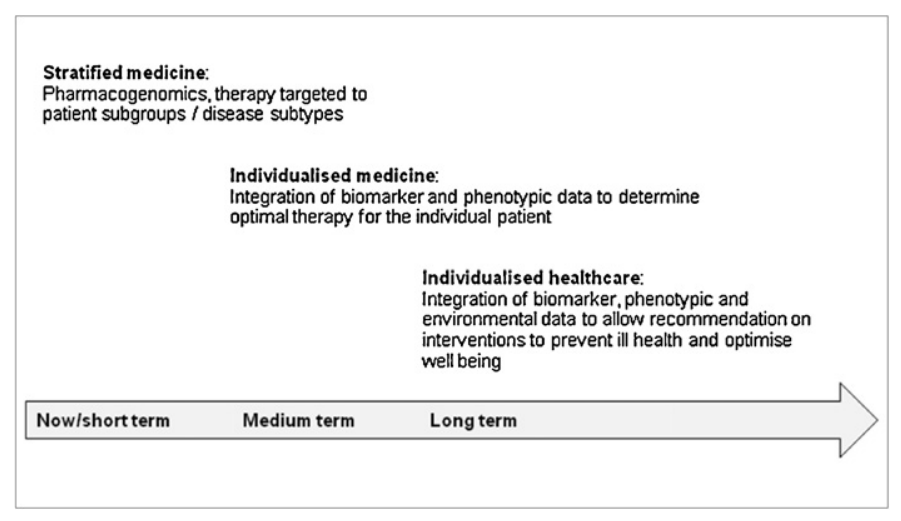

FIGURE 1

Personalised healthcare: from stratified medicine to individualized healthcare. 


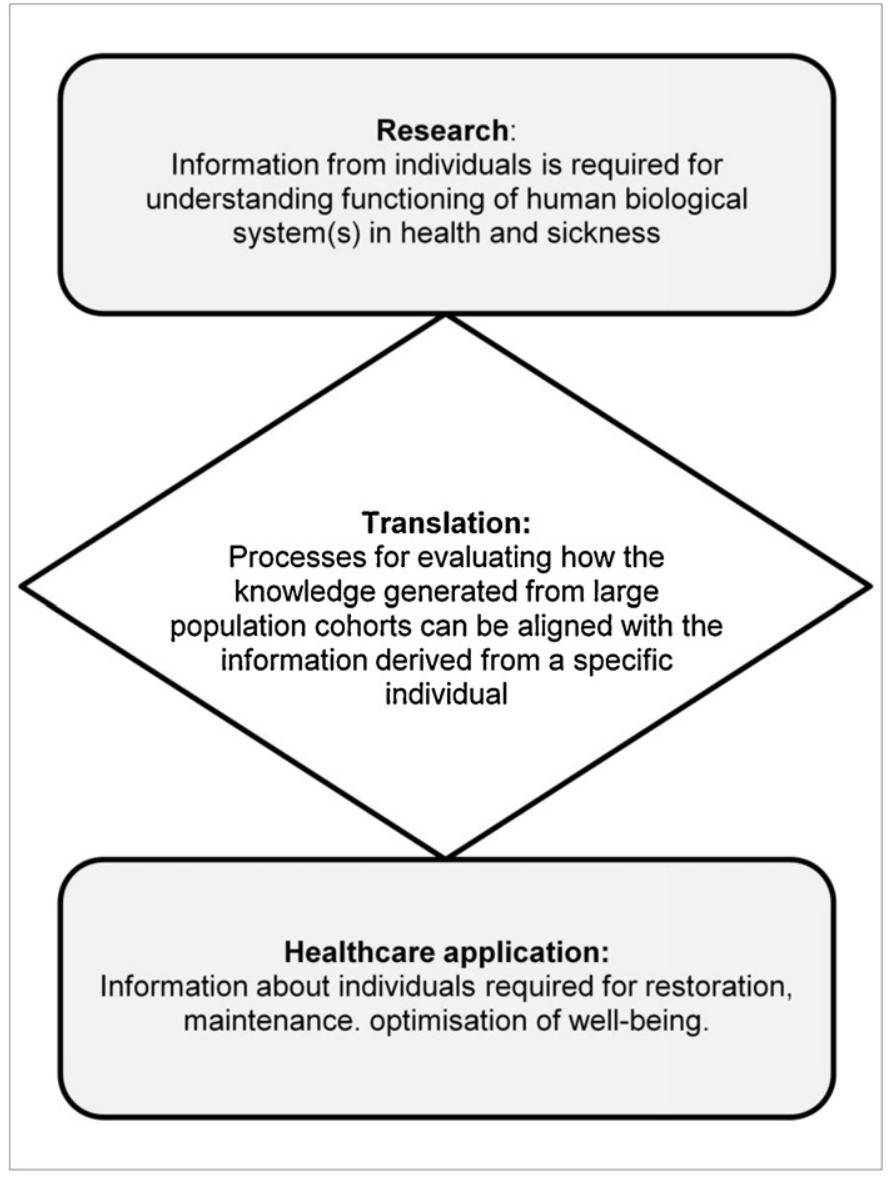

FIGURE 2

Translational process from basic research to healthcare interventions.

organism functions as a dynamic entity, with the system in flux as it interacts with its environment over time.

\section{Turning 'more information' into 'personalised medicine' \\ Research}

Central to the research into human biological variation that is underpinning the drive towards personalised medicine is the collection of biological, clinical, environmental, lifestyle and other data from large numbers of individuals with shared

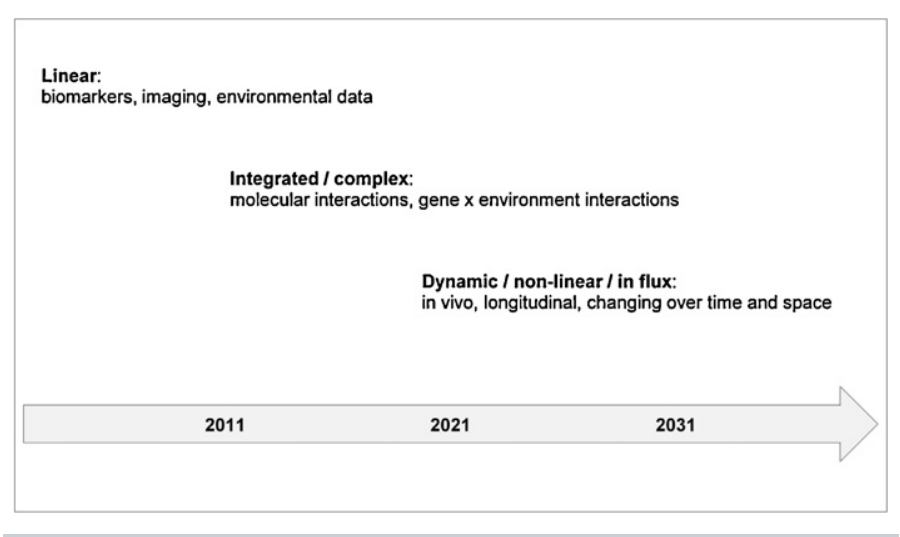

FIGURE 3

Types of information underpinning personalised medicine. characteristics (cohorts). The repositories for these data sets are commonly referred to as 'biobanks', and such biobanks have been set up in several European countries. These biobanks are a valuable resource in themselves and represent essential resources in advancing our understanding of the complex biology that underlies personalised medicine. However, for scientist working in different research sites, the ability to draw on data from multiple biobanks is key to moving research forward speedily and efficiently. Pooling data would maximise the utility of the biobanks, optimising return on the not inconsiderable investment made in their establishment and curation. A key requirement is standardisation of data, so that it is collected in uniform format across different locations and at different times. Steps are being taken towards a framework for standardisation of biobanks data within Europe, through the Biobanking and Biomolecular Resources Research Infrastructure (http://www.bbmri.eu/), and a proposal for a functional pan-European biobank has been presented to Member States of the European Union for approval and funding. Recommendations specific to this area are outlined in the ESF Position Paper European Biobanks and sample repositories - relevance to Personalised Medicine that was published in May 2011.

There are potential sources of research material in addition to biobanks. A wealth of data is starting to be produced outside traditional biomedical research and development settings. Citizens are starting to generate information on their biological status for themselves through 'self tracking' of their physiological, environmental and lifestyle variables, facilitated by initiatives such as 'Quantified Self' (http://quantifiedself.com/). There are examples where the data generated by the citizen-as-consumer for their own benefit are made available for research, as with the genetic test provider '23andMe' (https://www.23andme.com/) which offers those who purchase its tests the opportunity to share their information with its researchers. However, there are ethical and legal questions concerning ownership that need to be addressed if the data citizens produce on themselves for their own use is to be made available to the wider research community, allowing a private resource to be brought into the public domain for public good. There are also technical issues concerning the natures of citizengenerated data, and whether it can be made available in the standardised format required by researchers.

In addition to standardisation, a further requirement of the scientific community lies in the accurate and precise quantification of data to allow inputs from different platforms to be merged for maximum effect. Biological imaging data may pose a particular challenge in this respect. If wider types of data (lifestyle, environmental) are to be included in analyses then further difficulties are likely to arise. Capturing the clinical phenotype with all its complexity that includes environmental, economic and family circumstances is a daunting challenge. Such 'phenomes' also incorporate deep phenotyping so that maximum knowledge about an individual's disease can be used to interface with pathways of disease identified through 'omics' technologies. The informatics challenge here is far from trivial, not least capturing sufficient detail about the phenome.

Producing suitably standardised and quantified linear data is likely to require new techniques or technologies. As noted, generation of data on integrated biological systems is as yet at an early stage, and producing data on dynamic systems is yet to be 
addressed; again, new technologies will be necessary. Data analysis makes further technological demands. Access to increased IT capacity - both storage capacity and data processing capacity is going to be needed for processing vast quantities of linear data. New algorithms will also facilitate data processing. Integration of multiple data types will require new tools, as will handling individual longitudinal data from highly dynamic systems changing over time and space.

\section{Translation}

The first challenge for bioscience research is to collect large amounts of standardised data and process it into biological information and hence into enhanced understanding of human functioning in health and disease. The next challenge is to use this enhanced understanding of biology to optimise interventions that restore or maintain health on a person-specific basis. The usefulness of such interventions must be proven before if they are to become part of healthcare practice. A further key challenge is to develop methodologies for assessing the effect of interventions in the realm of personalised medicine.

Two broad questions need to be addressed as knowledge is translated from medical science to healthcare practice. The first concerns validity: is the knowledge generated from large population data sets relevant when applied to the individual? The second concerns utility: does application of knowledge lead to improvement in wellbeing? Utility has been defined in medical terms: is there an improvement in individual or population health? However, there are calls for incorporating measures of 'personal utility' in assessing personalised healthcare interventions $[11,13]$. The person may find the intervention useful, even if it does not lead to improved health. Empirical research has shown that for some people the mere fact of being better informed - regardless of the actionability of this information - is felt to be a benefit. For example, a diagnostic test can allow the person to better prepare for the future, even when there is no cure for the disease [14].

While the randomised controlled trial has become the gold standard for evidence in biomedicine, it is likely that personalised medicine will require the acceptance of a more 'portfolio' evidence base, encompassing retrospective analyses, prospective studies and comparative effectiveness studies alongside RCTs [5: 210]. It has also been suggested that it is important to balance the demand for robust evidence of effectiveness against the costs of delaying implementation. Others have been more forthright, arguing that personalised medicine poses a challenge to the dominance of Evidence Based Medicine, or at least will force EBM to confront internal questions about what it is, and about its principles of operation [15].

Certainly, the concept of 'personal utility', which is wider than 'clinical utility', challenges existing frameworks for assessing effectiveness of healthcare interventions. An intervention has personal utility if, for example, it makes a patient feel happier (before or without this increase in happiness necessarily being quantified and correlated with specific health outcomes), or if it enables a more active or empowered involvement of a patient in decisions pertaining to her health. Developing measures for assessing personal utility would require more input from patients/citizens themselves. Already, it is clear that the relationships between patients/citizens and researchers are changing. For a long time, the field of medicine had been characterised by a dividing line between knowledge producers and experts (scientists, researchers, technology developers, among others) on the one hand and knowledge recipients, or 'lay people' (patients, patients' family members, among others) on the other. With the increasing importance of patient advocacy groups in instigating, funding, and coordinating research at the end of the last century [16-18], the boundary between the two had become increasingly blurred. More recently, a much broader range of initiatives and activities of patients and citizens have moved into the spotlight of scientific and popular journals: Labelled 'garage biologists' [e.g. 19], or 'citizen scientists', these individuals and groups have contributed considerable achievements in a domain which had previously been inhabited by professional experts only. For example, in spring 2011, members of the social network 'Patients Like $\mathrm{Me}^{\mathrm{\prime}}$ published results of a study of the effect of lithium carbonate on the progress of Amyotrophic Lateral Sclerosis (ALS) in Nature Biotechnology [20]. 149 ALS patients in the 'Patients Like Me' network had organised the study on their own after the publication of findings from a study based on a much smaller patient sample suggesting that lithium carbonate, which is commonly used as a mood stabiliser, could slow down the progress of ALS [21].

Further, we need to remember that the vision of 'personalised medicine' encompasses its application in different settings, addressing different facets of healthcare. As is already becoming a reality with pharmacogenetic testing, the personalised approach can be a matter of predicting the outcome of interventions to allow the selection of optimal therapy for that patient at that moment. In the future, it could be used to predict a person's risk of ill health, and guide the selection of ameliorative action. Establishing evidence of effectiveness for preventive action is particularly challenging, as has been recognised even by those who insist on the need for such evidence [see, e.g. 22: 123].

There are then significant questions about appropriate methodologies for evaluating validity and utility that will need to be addressed to move personalised medicine from the lab into healthcare. While providing suitable answers to questions sufficient for introduction of a new tool into healthcare in principle, in practice a further question needs to be addressed, that of cost effectiveness. This last is one that personalised medicine is well positioned to answer. Indeed, one of the main benefits put forward by proponents of personalised medicine is that it will reduce costs by better targeting interventions to the individual, avoiding expenditure on interventions that will be ineffective for that patient, or cause adverse effects necessitating further medical input ('precision medicine').

\section{Healthcare applications}

In healthcare, personalised medicine involves the collecting of information from the individual to determine the optimum therapy or best preventive strategy. This will, at least in its first stages, involve turning a research technique into a tool for identifying and/or measuring a biomarker or set of biomarkers that can be used as routine within healthcare practice.

Again, it must be remembered that personalised medicine spans application in different contexts, by different users, and the form that that tool needs to take will vary. As it has thus far been realised, personalised medicine takes place in a clinical context, 
and involves guiding the clinician's decision on preventative approaches and drug therapy (pharmacogenomics). Developments in pharmacogenomics are spurring a burgeoning industry in production of companion diagnostics: biomarker assays that are developed alongside the therapeutic product and are used to determine the efficacy and/or safety of that drug for a particular patient. Pharmaceutical companies themselves are opening companion diagnostics divisions (Roche), and diagnostics companies are partnering with pharmaceutical companies to develop companion diagnostics for their drugs (Qiagen + Pfizer). Thus far, these companion diagnostics entail taking a sample from the patient and sending it to the laboratory for analysis with specialized equipment. However, even in genetic testing there is a move towards near-patient diagnostics or point-of-care testing, bringing the test to the patient. As an example of this, a hand-held device (the 'SNIP doctor') for analysing single nucleotide polymorphisms has been reported as successful in pilot studies (http://www.dnae.co.uk/news.asp). If personalised medicine is not to buck this trend, it will need to look to producing diagnostic modalities in the form of kits, machines or portable imaging devices that are easy to use for the health professional.

In the longer term, personalised medicine may come to involve citizens being health literate and using tools to monitor and manage their health status as part of their everyday life [23]. Self-monitoring is not new, of course: it is a central strategy for management for chronic conditions such as diabetes or asthma. There has also been a recent proliferation in commercial availability of tools for self-monitoring, such as blood pressure monitoring devices now for sale in the high street pharmacy. Novel technologies are being employed to facilitate health monitoring, for example the creation of an iPhone app to track food intake via product barcodes (dailyburn.com/foodscanner). Devices are becoming more sophisticated, capable of tracking several physiological variables and communicating them to a mobile smart phone or other computerised device. For example, the 'raisin personal monitor' produced by Proteus Biomedical can monitor and analyse heart rate, physical activity, body position and patient-logged events, and is worn like a sticking plaster or as an implant under the skin (http://www.proteusbiomed.com/ 2010/09/03/the-raisin-system/). Alongside this monitoring device, Proteus is developing 'chip-on-a-pill' technology, or ingestible event markers: ingestible microsensors that can be embedded in tablets or pills. Currently presented as a way of monitoring, and encouraging, patient adherence to medication, such markers could be used to feed back data from diagnostic or other measuring devices on or in the person. These are the sorts of technologies and gadgets that will allow the ongoing monitoring of functional status in real time, allowing fine tuning of therapy to control chronic disease, or adjustment of lifestyle to achieve the person's health goals.

\section{Factors affecting the development of technologies for personalised medicine Integration}

At one level, integration is a technical matter. It is the bringing together of different sorts of information - biological, clinical, environmental, lifestyle - about the participant in a research study or the patient in the clinic or the citizen striving to optimise their health. This bringing together must move from the current linear, cumulative model to a truly integrative model that can generate system-level knowledge $[24,25]$. At another level, what is required is organisational integration. There needs to be an integrated framework for doing science, within and across countries in the EU and beyond, making it possible for scientists in different research sites to work together on the same problems. Just as at a technical level, integrating data are distinct from accumulating data, so organisational integration means a step change from organisational co-ordination. It demands more than an environment where different research groups work on different aspects of the same problem and then pool their endeavours; it means a common research infrastructure that promotes synergistic working.

Integration also requires attending to the different sites where knowledge can be produced and the different actors that may have a role to play. As noted earlier, the dividing line between knowledge producers and experts on the one hand, and knowledge recipients and lay people on the other, is no longer tenable in the field of bioscience and health. Recently, computer gamers solved a puzzle that scientists had been pondering for many years, namely how a particular HIV-related protein was structured. By playing a computer game called Foldit (fold.it), citizens had determined the crystal structure of M-PMV retroviral protease by producing an accurate model [26]. Power, agency and expertise are being redistributed in new ways $[27,28]$, and integration between the different sites of knowledge production will be important as personalised medicine develops.

At a further level, for effective translation between bench and bedside (a movement that is bi-directional) $[29,30]$ we need to address cultural integration between science and healthcare. In the past, the model for translation between science and medicine has revolved around the figure of the clinician-scientist, bestriding both camps research [31-33]. In this era where science is getting bigger and medicine more person-specific, reliance on this colossus for effective and timely translation is becoming increasingly untenable. Of course, scientists and clinicians can and do communicate, but new effort and new initiatives may be needed to foster integration between research (encompassing both academia and industry) and healthcare practice.

\section{Harmonisation}

To produce the standardised data that will speed up research, there needs to be harmony in the way that data are collected, checked for accuracy and stored. Practical measures for implementing this harmonisation entail standard operating procedures for data gathering and data processing that are enforced and backed by quality assurance monitoring. Such procedures should be applied to all types of data (biological, clinical, environmental) and should extend to include information on the provenance of that data. Moving beyond data collection and data analysis, if research output is to be integrated as suggested above, there needs to be harmonisation of ways of doing the research to turn information into knowledge of biological functioning and to turn knowledge of biological functioning into interventions to alter that functioning in favour of health.

Important for harmonisation will be more effective tools for communication, between different sciences and between science 
and medicine/healthcare. The different branches of science working in systems biology (such as 'wet lab' scientists, informatics experts, physicists, engineers and mathematicians) and in other areas impacting on personalised medicine need to learn each other's languages, not fluently, but enough to work together on a common problem. The indications are that scientists have recognised and are addressing this matter [34,35]. A more effective dialogue between bioscience and biomedicine about what are the health problems that need addressing and what sort of solutions it is possible to implement in practice will ensure that research is targeted to priority areas and produces workable outputs. Alongside this, doctors and other health professionals will need education about the technologies that are developed for personalised medical and public health practice.

A further facet of harmonisation is the development of a common regulatory framework for the issues that impact on personalised medicine, across the EU and beyond. At one end of the spectrum of activities in personalised medicine, there is currently a lack of harmony in regulations pertaining to data gathering and data storage, such as how participants are enrolled in biobanks, how their personal information may be stored and used. At the other end of the spectrum, the development of new in vitro diagnostic tests creates demands for new regulations. Pharmacogenomics posed a problem for pre-existing regulatory frameworks, combining as it does approval of diagnostics and therapeutics. The lack of clear regulatory standards for pharmacogenomic testing has been widely cited as a barrier to its introduction [36]. The absence of pre-existing regulations may provide an opportunity to build harmonisation in from the start, but this will be challenging. Some efforts such as those of the Public Health Genomics European Network (PHGEN), however, are underway in this area that may assist Member States, Applicant and EFTA-EEA countries with evidence-based guidance $[37,38]$.

Alongside the development of appropriate regulatory frameworks, bioethical norms and guidelines will need to accommodate the changing relationships between knowledge producers and knowledge recipients in the field of health and medicine. For example, instances such as the aforementioned example of citizens organising their own clinical studies, or contributing genotypic and phenotypic information for genetic association research via commercial online genetic testing services [39], raise questions about the need for new instruments and institutional landscapes for research ethics (e.g. what sort of ethics approval is required when research and science takes place outside of formal institutions and organisations? Who could and should be entrusted with giving ethics approval to bottom-up research studies? among others). With regard to large-scale data collections, bioethical challenges continue to exist regarding data protection, confidentiality and privacy; genetic discrimination; ownership and return on investment (including access to findings). It becomes increasingly apparent, however, that the notion of data confidentiality and privacy will need to be redefined in the era of data-rich medicine, where online databases will become the crucial platform for decision-making in health. In addition, current requirements for biobank-based research in personalised medicine encounter strict requirements regarding (re-)consenting individuals which have been criticised as unduly impeding research $[40,41]$.
It will also be important to consider whether personalised medicine is in harmony with the wider culture in which it is developing. What are the views of patients, publics and policy makers? It will be necessary to engage in dialogue with patients or citizen-consumers of personalised medical services, with health service commissioners and with policy makers as personalised medicine is taken forward, to ensure that everyone is 'singing from the same hymn sheet'. This does not mean bending everyone to the will of personalised medicine; it is as much a matter of ensuring that personalised medicine does not expend wasted effort on lines of enquiry that are not practicable, not welcome, or not tenable in the prevailing political climate. The philosophy of personalised medicine has thus far been very much in tune with the political will in the liberal democracies in which it has been most advanced. In such nations, 'personalisation' has a currency in policy circles that goes well beyond 'personalised medicine' [e.g. 42]. Principles of increasing citizens' choice, personalising services to the user, and involving lay-people in decision making have become central in much policy making, in areas well beyond healthcare. However, at the level of local implementation of policy, and in different cultural or political contexts, there may be less synergy with existing ways of thought and practice.

\section{Resources}

Inevitably, resources are an issue for the development of technologies for personalised medicine. Researchers need access to the necessary computing resources for data processing and data storage. This may require re-allocation of funding within bioscience or re-prioritisation of bioscience by funding bodies such as national governments to ensure access to IT resources that already exist. It may also require the development of novel data processing tools that could provide a step change in capacity, in the same way that the development of next-generation technologies have allowed cheaper, faster DNA sequencing. The right researchers are also needed. There is a concern at lack of people with the requisite skills and expertise. They need to be trained, and they need to be attracted to work in the field.

More widely, there needs to be sufficient funding for both basic and translational research in the field. The development of new technologies for personalised medicine and their introduction into healthcare practice will necessitate new business models for the pharmaceutical industry as the emphasis shifts from blockbuster drugs, the exploitation of new opportunities by in vitro diagnostics firms, and new partnerships between the two. It has been argued that translational research is chronically underfunded [43], but basic research is coming under increasing pressure. The promises of personalised medicine entail revolutionary as well as evolutionary developments: moving from linear to integrated and dynamic data, from elucidating the operation of biological components to understanding the functioning of biological systems, from a one-size-fits-all healthcare based on what is best for the average patient to a healthcare that is optimised for the specific individual. These shifts demand a true creativity that is properly inventive, rather than new arrangements of what already exists [44]. Such creativity requires not only enormous hard work, but also a willingness to be frustrated. As the philosopher Gilles Deleuze has described 
it: 'A creator who isn't seized by the throat by a set of impossibilities is no creator. ... It's by banging your head against the wall that you find an answer' [45: 292]. Scientists may already be willing to 'bang their heads against the wall' in their efforts to develop new technologies for collecting and analysing integrated and dynamic data, new methodologies for evaluating the effectiveness of interventions based on knowledge of system level functioning and new tools for remote real time monitoring of health status. Funding agencies must be convinced of the need for such sort of 'disruptive research' that allows them to do so.

\section{Technologies, health and harmonisation}

If harmonisation between biomedicine and society is to be ensured, then personalised medicine must provide social benefits. Sociologists have charted a contemporary preoccupation with avoiding death as a modern strategy for achieving immortality such that 'our expectations - the ways in which we are shaping our hopes for salvation, for the future for ourselves - are themselves shaped by considerations about the maintenance of health and the prolongation of earthly existence' [46: 258]. As such, death can be 'forgotten in the daily bustle about health' [47: 19]. Biomedicine has been extraordinarily effective at producing new technologies that meet the demand for prolonged life. However, decreases in mortality have come at the expense of health; morbidity levels have increased as what were once fatal illnesses are turned into chronic diseases. There are signs of a cultural shift away from an overriding pre-occupation with avoiding death. We may not yet have overcome the cultural iatrogenesis that has undermined culturally specific ways of dealing with sickness and death as part of human experience [48: 41-42], but the introduction of 'living wills' and the movements for legalisation of physician-assisted suicide evidence a growing questioning of the 'life at all costs' philosophy. In this respect 'the primary goal of personalized medicine should be to increase the quality of life first, and lifespan second' [49: 1977], and personalised medicine must avoid introducing new technologies into healthcare before or unless they can improve wellbeing.

'Wellbeing' is a fluid concept. A current advertising campaign by the UK's leading private health provider has the strap-line 'healthy means different things to different people' (http://findhealthy.bupa.co.uk/, http://www.youtube.com/watch?v=iZGSi_k9ne4). Personalised medicine should, in theory, be well positioned to embrace the concept of health as a personal optimum of functioning, rather than a species standard. However, in practice this will not be without its challenges. Medicine has distinguished between health and disease, normal and pathological, using biomarkers that indicate status at a point in time relative to the species standard. Shifting away from the 'average' and towards examining functioning over time raises questions about how to define 'normal' or 'healthy' (and hence 'abnormal' or 'unhealthy').

At one level, this is a technical challenge, to measure the level of normal (healthy) inter- and intra-individual variability in levels of key biomarkers. Research has started to address this problem [50-52], but it is an area where more input is likely to be necessary. Furthermore, the non-linear and network-based

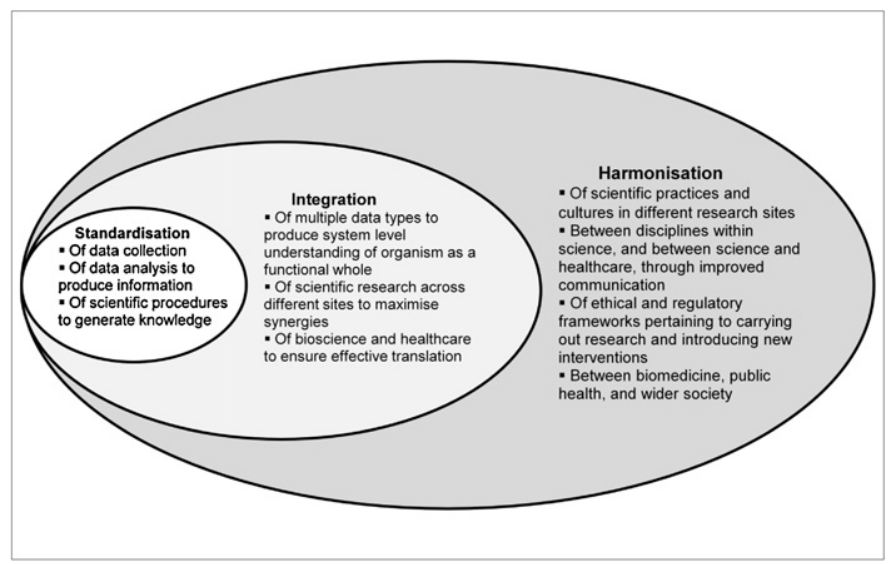

FIGURE 4

Standardisation, integration and harmonisation in personalised medicine.

research is already starting to change disease nosology. Seemingly dissimilar diseases and health outcomes are being lumped together based on common pathways. What were thought to be single diseases are being split into separate ailments ('diseasomes') belonging together such as rheumatoid arthritis and B-cell lymphoma. Thus, we face a time when not only the taxonomy of human disease is being redefined given the existence of pathological and molecular disease subtypes, but also when boundaries of disciplines are crossed and the understanding of diseases is changed as it happened before with the jump from the macroscopic view in anatomy to the microscopic view in cell structure [11].

There is also a question starting to be asked in science as to whether a definition of health as presence or absence of a specific biomarker or group of biomarkers is appropriate in the era of systems biology. An alternative that is being proposed involves understanding health in terms of system robustness, that is, the ability of the biological system to maintain homeostasis in the face of perturbations [53-57]. Looking beyond technological development over the next 20 years, in the future will we understand health in a different way? If so, will this have led to different sorts of technologies for determining and for modifying functional status?

\section{Conclusion}

The key issues for the future of technologies for personalised medicine that emerged from the ESF workshop were standardisation, integration and harmonisation (Fig. 4).

In the medium term, the application of various '-omics' techniques is likely to lead to more individualised treatments. Further development of existing technologies and the invention of new ones will be vital for progress in both the science of personalised medicine and the healthcare practices that derive from it. However, factors surrounding the technologies have a crucial impact on the trajectory that is taken, on how and indeed whether these technological developments are made possible. The ESF Forward Look is addressing these issues in several 'big picture' workshops that will consider the wider context in which personalised medicine is coming to fruition. 


\section{References}

1 Hood, L. et al. (2004) Systems biology and new technologies enable predictive and preventative medicine. Science 306, 640-643

2 Abrahams, E. (2008) Right drug, right patient, right time: personalized medicine coalition. Clin. Transl. Sci. 1, 11-12

3 Galas, D. and Hood, L. (2009) Systems biology and emerging technologies will catalyze the transition from reactive medicine to predictive, personalized, preventive and participatory (P4) medicine. Interdiscip. Biol. Central 1, 0006

4 Ginsburg, G.S. and Willard, H.F. (2009) Genomic and personalized medicine: foundations and applications. Transl. Res. 154, 277-287

5 Green, E.D. and Guyer, M.S. (2011) Charting a course for genomic medicine from base pairs to bedside. Nature 470, 204-213

6 Berg, M. (1995) Turning a practice into a science: reconceptualizing post-war medical practice. Soc. Stud. Sci. 25, 437-476

7 Sackett, D. et al. (1996) Evidence-based medicine: what it is and what it isn't. Br. Med. J. 312, 71-72

8 Timmermans, S. and Berg, M. (2003) The Gold Standard: The Challenge of Evidencebased Medicine and Standardization in Health Care. Temple University Press, Philadelphia

9 Ferraldeschi, R. and Newman, W.G. (2011) Pharmacogenetics and pharmacogenomics: a clinical reality. Ann. Clin. Biochem. 48, 410-417

10 Hedgecoe, A. (2004) The Politics of Personalised Medicine: Pharmacogenetics in the Clinic. Cambridge University Press, Cambridge

11 Brand, A. (2011) Public health genomics - public health goes personalized? Eur. J. Public Health 21, 2-3

12 Editorial, (1999) Complicated is not complex. Nat. Biotechnol. 17, 511

13 Khoury, M.J. et al. (2009) The scientific foundation for personal genomics: recommendations from a National Institutes of Health-Centers for Disease Control and Prevention multidisciplinary workshop. Genet. Med. 11, 559-567

14 Green, R.C. et al. (2009) Disclosure of APOE genotype for risk of Alzheimer's disease. N. Engl. J. Med. 361, 245-254

15 Miles, A. et al. (2008) Evidence-based healthcare, clinical knowledge and the rise of personalised medicine. J. Eval. Clin. Pract. 14, 621-649

16 Rabeharisoa, V. and Callon, M. (2002) The involvement of patients' associations in research. Int. Soc. Sci. J. 171, 57-65

17 Novas, C. (2006) The political economy of hope: patients' organizations, science and biovalue. BioSocieties 1, 289-305

18 Panofsky, A. (2011) Generating sociability to drive science: patient advocacy organizations and genetics research. Soc. Stud. Sci. 41, 31-57

19 Anon., (2010) Garage biology. Nature 467, 634

20 Wicks, P. et al. (2011) Accelerated clinical discovery using self-reported patient data collected online and a patient-matching algorithm. Nat. Biotechnol. 29, 411414

21 Fornai, F. et al. (2008) Lithium delays progression of amyotrophic lateral sclerosis. Proc. Natl. Acad. Sci. U. S. A. 105, 2052-2057

22 Wanless, D. (2004) Securing Good Health for the Whole Population. HM Treasury, London

23 Brand, A. and Brand, H. (2011) Health literacy and public health genomics: innovation management by citizens: editorial. Public Health Genomics J. 14, 193194

24 Huang, S. (2000) The practical problems of post-genomic biology. Nat. Biotechnol. $18,471-472$

25 Kitano, H. (2002) Looking beyond the details: a rise in system-oriented approaches in genetics and molecular biology. Curr. Genet. 41, 1-10

26 Khatib, F. et al. (2011) Crystal structure of a monomeric retroviral protease solved by protein folding game players. Nat. Struct. Mol. Biol. 18, 1175-1177

27 Prainsack, B. et al. (2008) Misdirected precaution. Nature 456, 34-35

28 Prainsack, B. (2011) Voting with their mice: personal genome testing and the participatory turn in disease research. Account. Res. 18, 132-147

29 Marincola, F. (2003) Translational medicine: a two-way road. J. Transl. Med. 1
30 Wainwright, S.P. et al. (2006) From bench to bedside? Biomedical scientists' expectations of stem cell science as a future therapy for diabetes. Soc. Sci. Med. 63, 2052-2064

31 Carrel, T. (2002) The relationship between surgeon and basic scientist. Transpl. Immunol. 9, 331-337

32 Sartor, R.B. (2003) Translational research: bridging the widening gap between basic and clinical research. Gastroenterology 124, 1178

33 Archer, S.L. (2007) The making of a physician-scientist-the process has a pattern: lessons from the lives of Nobel laureates in medicine and physiology. Eur. Heart J. $28,510-514$

34 Calvert, J. (2010) Systems biology, interdisciplinarity and disciplinary identity. In Collaboration in the New Life Sciences (Parker, J.N., Vermeulen, N., Penders, B., eds), Ashgate, Aldershot

35 Lewis, J. (2010) Matchmaking mechanisms: collaborative arrangements in proteomics and bioinformatics. In Collaboration in the New Life Sciences (Parker, J.N., Vermeulen, N., Penders, B., eds), Ashgate, Aldershot

36 Hogarth, S. (2011) Regulatory experiments and transnational networks: the governance of pharmacogenomics in Europe and the United States. Global Biopolitics Research Group: Working paper 36. (http://www.kcl.ac.uk/sspp/ departments/politicaleconomy/research/biopolitics/publications/ workingpapers/workingpapers31-37.aspx; accessed 20.10.11).

37 Morre, S.A. et al. (2009) The EU FP6 EpiGenChlamydia Consortium: contribution of molecular epidemiology and host-pathogen genomics to understanding Chlamydia trachomatis-related disease. Drugs Today 45, 7-13

38 Rosenkötter, N. et al. (2010) The contribution of health technology assessment, health needs assessment, and health impact assessment to the assessment and translation of technologies in the field of public health genomics. Public Health Genomics 14, 43-52

39 Tung, J.Y. et al. (2011) Efficient replication of over 180 genetic associations with self-reported medical data. PLoS One 6, e23473

40 Taylor, P. (2008) When consent gets in the way. Nature 456, 32-33

41 Prainsack, B. and Buyx, A. (2011) Solidarity: Reflections on an Emerging Concept in Bioethics. Nuffield Council on Bioethics, London

42 Office of Public Services Reform, (2005) Putting People at the Heart of Public Services. Office of Public Services Reform, London

43 Pardridge, W. (2003) Translational science: what is it and why is it so important? Drug Discov. Today 8, 813-815

44 Osborne, T. (2003) Against 'creativity': a philistine rant. Econ. Soc. 32, 507-525

45 Deleuze, G. (1992) Mediators. In Incorporations (Crary, J. and Kwinter, S., eds), New York, Zone Books

46 Rose, N. (2006) The Politics of Life Itself. Princeton University Press, Princeton, NJ

47 Bauman, Z. (1992) Survival as a social construct. Theory Cult. Soc. 9, 1-36

48 Illich, I. (1977) Limits to Medicine (Medical Nemesis: The Expropriation of Health). Penguin, Harmondsworth

49 Sander, C. (2000) Genomic medicine and the future of health care. Science 287, 1977-1978

50 Whitney, A.R. et al. (2003) Individuality and variation in gene expression patterns in human blood. Proc. Natl. Acad. Sci. U. S. A. 100, 1896-1901

51 Eady, J.J. et al. (2005) Variation in gene expression profiles of peripheral blood mononuclear cells from healthy volunteers. Physiol. Genomics 22, 402-411

52 Dumeaux, V. et al. (2010) Deciphering normal blood gene expression variationThe NOWAC postgenome study. PLoS Genet. 6, e1000873

53 Carlson, J.M. and Doyle, J. (2002) Complexity and robustness. Proc. Natl. Acad. Sci. U. S. A. 99 (Suppl. 1), 2538-2545

54 Kitano, H. (2004) Biological robustness. Nat. Rev. Genet. 5, 826-837

55 Stelling, J. et al. (2004) Robustness of cellular functions. Cell 118, 675-685

56 Elliott, R. et al. (2007) Nutrigenomic approaches for benefit-risk analysis of foods and food components: defining markers of health. Br. J. Nutr. 98, 1095-1100

57 Kitano, H. (2007) Towards a theory of biological robustness. Mol. Syst. Biol. 3, 137 